\title{
Clinical efficacy of immune checkpoint inhibitors in the treatment of unresectable advanced or recurrent gastric cancer: an evidence-based review of therapies
}

This article was published in the following Dove Press journal: OncoTargets and Therapy

\author{
Kazuhiro Togasaki \\ Yasutaka Sukawa \\ Takanori Kanai \\ Hiromasa Takaishi \\ Department of Internal Medicine, \\ Division of Gastroenterology \\ and Hepatology, Keio University \\ School of Medicine, Shinjuku-ku, \\ Tokyo 160-8582, Japan
}

Correspondence: Yasutaka Sukawa Department of Internal Medicine, Division of Gastroenterology and Hepatology, Keio University School of Medicine, 35 Shinanomachi, Shinjuku-ku, Tokyo 160-8582, Japan

$\mathrm{Tel}+8 \mid 33533790$

Fax $+8 I 353636247$

Email sukawa@keio.jp

\begin{abstract}
Standard treatment options for patients with advanced gastric cancer (GC) offer limited efficacy and are associated with some toxicity, which necessitates the development of more effective therapies for improving the treatment outcomes for this disease. Immunotherapy involving immune checkpoint inhibitors (ICIs) which inhibit the programmed death 1 (PD-1)/ programmed death ligand 1 interaction has emerged as a new treatment option. Nivolumab, a human IgG4 monoclonal antibody inhibitor of PD-1, has demonstrated promising clinical activity and induced durable responses in patients with advanced GC. Nivolumab has recently been approved for treating patients with pretreated advanced GC in Japan. In the present review, we summarized current evidence of the clinical efficacy of ICIs in a variety of solid tumors and reported our experience in patients with GC who were treated with nivolumab and the interesting features that were observed in these cases. Certain ICI-specific clinical features such as pseudo- and hyper-progression of tumor and hyper-response to subsequent chemotherapy have been reported in several cancer types. Lastly, we discussed the present scenario regarding research on biomarkers for assessing the clinical benefits of ICI therapies.
\end{abstract}

Keywords: nivolumab, pembrolizumab, avelumab, pseudoprogression, hyperprogression, hypersensitivity, microbiome

\section{Introduction}

Gastric cancer (GC) is the fifth most frequently occurring cancer and is the third most common cause of cancer mortality worldwide. ${ }^{1}$ Clinical trials have demonstrated the effectiveness of conventional chemotherapy in treating patients with advanced GC. Particularly, anti-HER2 antibody trastuzumab and the antivascular endothelial growth factor receptor-2 antibody ramucirumab have been shown to provide survival benefits for patients with advanced GC, while the development of many other molecular targeted drugs has failed. However, the prognosis of advanced GC remains very poor, necessitating the development of novel drugs.

Recently, immune checkpoint inhibitors (ICIs) have revolutionized the treatment of various types of advanced cancers. ATTRACTION-2 (ONO-4538-12) was the first randomized Phase III trial which was designed for investigating the efficacy and safety of nivolumab, a human IgG4 monoclonal antibody inhibitor of programmed death 1 (PD-1), in heavily pretreated patients with unresectable or recurrent gastric or gastro-esophageal junction cancer (GC/GEJC). ${ }^{2}$ Compared with placebo, nivolumab 
achieved a higher efficacy in this study. Consequently, nivolumab has recently been approved in Japan for treating patients with pretreated advanced GC/GEJC. Also, pembrolizumab, another human IgG4 monoclonal antibody inhibitor of PD-1, and avelumab, human IgG1 monoclonal antibody inhibitor of programmed death ligand 1 (PD-L1), are undergoing clinical trials, although they have not been approved yet in Japan.

On the other hand, unusual ICI-specific clinical features, such as pseudo- and hyper-progression of tumor and hyperresponse to subsequent chemotherapy, have been reported in several types of cancers. However, data regarding ICIspecific features in GC are limited.

In this review, we summarized current evidence on ICI efficacy and safety along with interesting clinical features that we experienced while treating patients with GC. Additionally, we focused on ICI development in the treatment of GC and reviewed the present scenario of biomarker research associated with ICIs. This review summarizes literature searches from PubMed and data reported during major oncology conferences until July 2018. Informed consent or a substitute for it was obtained from patients for being included in this review.

\section{ICls and GCs: clinical evidence \\ Nivolumab}

The GC cohort of the Phase I/II CheckMate-032 trial revealed clinical benefits associated with using nivolumab alone or in combination with ipilimumab, a fully human IgG1 monoclonal antibody inhibitor of cytotoxic T-lymphocyte associated protein-4 (CTLA-4), in patients with chemotherapy-refractory advanced GC/GEJC. ${ }^{3,4}$ A total of 160 heavily pretreated patients ( $79 \%$ had $\geq 2$ pretreatment therapy lines) were enrolled into three subgroups. The primary end point was objective response rate (ORR). Subgroup 1 received $3 \mathrm{mg} / \mathrm{kg}$ of nivolumab monotherapy; ORR was $12 \%$ (95\% CI: 5-23) and the median overall survival (OS) was 6.2 months $(95 \%$ CI: 3.4-12.4). Combination therapies with different doses of nivolumab plus ipilimumab were also assessed. Subgroup 2 received $1 \mathrm{mg} / \mathrm{kg}$ of nivolumab plus $3 \mathrm{mg} / \mathrm{kg}$ of ipilimumab and subgroup 3 received $3 \mathrm{mg} / \mathrm{kg}$ of nivolumab plus $1 \mathrm{mg} / \mathrm{kg}$ of ipilimumab. In the subgroups 2 and 3, ORRs were 24\% (95\% CI: 13-39) and 8\% (95\% CI: 2-19), respectively, similar to ORRs in the monotherapy setting. Median OS rates were 6.9 (95\% CI: 3.7-11.5) and 4.8 (95\% CI: 3.0-8.4) months, respectively. Grade 3 or 4 treatment-related adverse events (TRAEs) were reported in $17 \%, 47 \%$, and $27 \%$ of patients in the subgroups 1-3, respectively. Responses were observed regardless of tumor PD-L1 status across the treatment subgroups. As a result, nivolumab and nivolumab plus ipilimumab demonstrated clinically meaningful efficacy and manageable safety profile, although combination therapy was not superior to nivolumab monotherapy.

On the basis of these results, the Phase III trial ATTRACTION-2 included patients with advanced GC/ GEJC who had been pretreated with $\geq 2$ chemotherapy regimens. ${ }^{2}$ In this randomized, double-blind, placebocontrolled, Phase III trial, 493 Asian patients were enrolled. Patients were randomly assigned (2:1) to intravenously receive $3 \mathrm{mg} / \mathrm{kg}$ of nivolumab or placebo every 2 weeks. OS was the primary end point in the intention-to-treat population. The median OS was 5.3 vs 4.1 months $(\mathrm{HR}=0.63$, 95\% CI: $0.49-0.75, P<0.0001)$, and ORR was $11 \%$ vs $0 \%(P<0.0001)$. The efficacy of nivolumab was observed irrespective of PD-L1 status (on the basis of a $\geq 1 \%$ cutoff in tumor cells). Notably, clear separation of the nivolumab and placebo OS curves occurred over time and was sustained beyond 1 year, which suggested a durable OS benefit with the use of nivolumab.

ICIs are associated with distinctive inflammatory adverse effects known as immune-related adverse events (irAEs), which can potentially affect any system in the body, but predominantly involve the skin, colon, lungs, endocrine glands, and liver. ${ }^{5}$ In the ATTRACTION-2 study, grade 3 or 4 TRAEs occurred in $10 \%$ of the patients treated using nivolumab compared with $4 \%$ of those in the placebo arm. All-grade TRAEs reported in $\geq 5 \%$ patients in the nivolumab group were pruritus, diarrhea, rash, and fatigue. The safety profile shared similarity with previous clinical trials for other tumor types. ${ }^{6-12}$ No GC-specific adverse events were observed.

\section{Pembrolizumab}

Pembrolizumab is another human IgG4 monoclonal antibody inhibitor of PD-1. In KEYNOTE-012 Phase I clinical trial, 39 patients with PD-L1-positive GC and chemotherapyrefractory metastatic disease were treated using pembrolizumab. Among these patients, eight (22\%) patients were judged to have had an overall response at central review. ${ }^{13}$ In the subsequent KEYNOTE-059 Phase II study, an unselected population of patients with GC $(n=133)$ who specifically received two pretreated lines showed an ORR of $16.4 \% .{ }^{14}$

On the basis of these results, KEYNOTE-061 Phase III trial compared pembrolizumab monotherapy with paclitaxel monotherapy in non-Asian patients with advanced PD-L1positive GC that had progressed following first-line platinum and fluoropyrimidine doublet therapy. ${ }^{15}$ Eligible patients were randomized (1:1) to receive either $200 \mathrm{mg}$ of pembrolizumab every 3 weeks for up to 2 years or standard-dose paclitaxel. 
The following two primary end points were considered: OS and progression-free survival (PFS) in patients with PD-L1 expression with combined positive score (CPS) of $\geq 1$. A total of 592 patients were enrolled; of the 395 patients who had PD-L1 CPS of $\geq 1,196$ patients were administered pembrolizumab and 199 patients paclitaxel. Median OS was 9.1 months with pembrolizumab (95\% CI: 6.2-10.7) and 8.3 months with paclitaxel (95\% CI: 7.6-9.0) ( $\mathrm{HR}=0.82$, 95\% CI: 0.66-1.03, $P=0.0421)$. The median PFS was 1.5 months with pembrolizumab (95\% CI: 1.4-2.0) and 4.1 months with paclitaxel (95\% CI: 3.1-4.2) (HR $=1.27,95 \%$ CI: $1.03-1.57)$. Grade $\geq 3$ TRAEs occurred in 42 (14\%) of the 294 patients treated with pembrolizumab and $96(35 \%)$ of the 276 patients treated with paclitaxel. Consequently, pembrolizumab did not significantly improve OS in comparison with paclitaxel as second-line therapy. In subgroup analyses, Eastern cooperative oncology group performance status (PS) 0, PD-L1 CPS $\geqq 10$, and microsatellite instability (MSI)-high were identified as predictive biomarkers for pembrolizumab. Together with the favorable safety profile, these data support further exploration for identifying patients who will likely benefit from pembrolizumab monotherapy and the ongoing development of pembrolizumab as part of combination therapy regimens for treating GC.

Recently, the US FDA granted approval of pembrolizumab for treating unresectable or metastatic MSI-high or mismatch repair deficient (dMMR) solid tumors progressing following prior treatment and in the absence of satisfactory alternative treatment options. This approval was granted on the basis of data obtained from 149 patients with MSI-high or dMMR cancers enrolled across five single-arm clinical trials. ${ }^{16-20}$ Ninety patients had colorectal cancer (CRC) and the remaining 59 patients had 1 of the 14 other types of solid tumors. The ORR with the use of pembrolizumab was $39.6 \%$ (95\% CI: 31.7-47.9), including 11 (7.4\%) complete response and 48 (32.2\%) partial response (PR). The median duration of response was not reached (range: 1.6-22.7 months). Among the patients who responded to pembrolizumab treatment, responses of $78 \%$ lasted for a minimum of 6 months. The findings of these studies resulted in the unprecedented FDA approval of pembrolizumab in treatment-refractory MSI-high solid tumors on the basis of tumor biomarkers, regardless of tissue histology.

\section{Avelumab}

Avelumab is a fully human anti-PD-L1 IgG1 monoclonal antibody. JAVELIN Gastric 300 Phase III trial compared avelumab treatment with the physician's choice of chemotherapy (either using paclitaxel or irinotecan) performed in 371 patients with advanced GC/GEJC as a third-line therapy, unselected for PD-L1 expression. ${ }^{21}$ The trial did not meet its primary end point of OS improvement (4.6 vs 5.0 months, $\mathrm{HR}=1.1,95 \% \mathrm{CI}: 0.9-1.4, P=0.81)$ or the secondary end points of PFS (1.4 vs 2.7 months, HR $=1.73,95 \%$ CI: $1.4-2.2$, $P>0.99)$ or ORR (2.2\% vs $4.3 \%)$ in the avelumab treatment vs chemotherapy arms, respectively. Grade $\geq 3$ TRAEs were noted in 17 patients $(9.2 \%)$ in the avelumab arm and in 56 patients $(31.6 \%)$ in the chemotherapy arm.

JAVELIN Gastric 100 (NCT02625610) trial compares maintenance therapy with avelumab vs continuation of firstline chemotherapy in patients with advanced GC/GEJC who have not shown progression following 12 weeks of oxaliplatin plus fluoropyrimidine chemotherapy. ${ }^{22}$ The hypothesis is that avelumab provides durable antitumor response following immunogenic priming and tumor shrinkage induced by first-line chemotherapy with the added benefit of avoiding the burden of toxicity from additional chemotherapy or combination therapy. The primary end points are OS and PFS, and recruitment has been completed.

\section{Combination therapy}

Two ongoing Phase III trials assessing ICI-based combination therapy as the first-line therapy are KEYNOTE-062 $\left(\right.$ NCT02494583) $^{23}$ and CheckMate 649 (NCT02872116). ${ }^{24}$ KEYNOTE-062 assesses the efficacy of pembrolizumab alone or in combination with cisplatin/5-fluorouracil (5-FU) vs cisplatin/5-FU alone in patients with PD-L1+/HER2advanced GC/GEJC. CheckMate 649 is a three-arm trial assessing the efficacy of nivolumab plus ipilimumab vs nivolumab plus capecitabine plus oxaliplatin (XELOX) or 5-FU plus oxaliplatin (FOLFOX) vs the investigator's choice of XELOX or FOLFOX in patients with previously untreated advanced or metastatic GC/GEJC. The primary end point in both studies is OS in patients with PD-L1-positive tumors.

ATTRACTION-4 (NCT02746796) is a Phase II/III trial that evaluated the safety and efficacy of nivolumab in combination with S-1 plus oxaliplatin (SOX) therapy or XELOX therapy as the first-line chemotherapy for Asian patients with unresectable advanced or recurrent GC/GEJC. ${ }^{25}$ It reported the interim safety and clinical activity data in 39 patients from part 1 of the study. The ORR for patients treated with nivolumab/SOX and nivolumab/XELOX was $67 \%$ and $71 \%$, respectively. Grade $\geq 3$ TRAEs, most of which corresponded with frequently occurring side effects of chemotherapy, occurred in $52 \%$ and $67 \%$ of the patients, respectively, and no difference was noted in activity and safety between these two regimens. Part 2 of this study, a randomized comparison of nivolumab vs placebo in combination with SOX/XELOX, is ongoing, and the results are awaited. 
NivoRam (NCT02999295) is a Phase I/II trial that evaluated the safety and tolerability of addition of ramucirumab to nivolumab in patients with GC as second-line therapy. ${ }^{26}$ The primary end points are dose-limiting toxicity for Phase I and 6-month PFS for Phase II. Preliminary results were recently reported and no new safety signals were noted. These results demonstrated promising efficacy and the result of the primary end point are expected in the near future.

A Phase I/II trial was also designed for determining the recommended dose and for evaluating the efficacy, safety, and predictive biomarkers of the combination regimen of paclitaxel, ramucirumab, and nivolumab in patients with GC as second-line therapy (UMIN000025947). The primary end points include dose-limiting toxicity for Phase I and 6-month PFS for Phase II.

Ongoing Phase III trials in the neoadjuvant/adjuvant setting include ONO-4538-38 (NCT03006705), a trial of nivolumab in combination with S-1 or XELOX vs S-1 or XELOX alone in patients with resected GC/GEJC and KEYNOTE-585 (NCT03221426), a trial comparing pembrolizumab plus chemotherapy vs chemotherapy alone as neoadjuvant or adjuvant treatment for untreated patients with GC/GEJC.

Tables 1 and 2 summarize the previous Phase III trials and ongoing trials of ICIs in GC, respectively.

\section{Clinical positioning of ICls in GC}

For patients with advanced or metastatic GC/GEJC who have good PS, combination therapy of fluoropyrimidine and platinum is the standard first-line therapy. Patients who have HER2-positive tumors should receive trastuzumab combined with fluoropyrimidine plus platinum. As secondline chemotherapy, paclitaxel combined with ramucirumab is recommended. This recommendation is on the basis of the results of the RAINBOW study, which showed the survival benefit of paclitaxel plus ramucirumab compared with paclitaxel alone. ${ }^{27}$ Since trastuzumab did not show a clear survival benefit beyond progression in the T-ACT study, ${ }^{28}$ continuing anti-HER2 therapy is not recommended for second-line chemotherapy following first-line chemotherapy with trastuzumab.

On the basis of the findings of the ATTRACTION-2 study, the Japanese Ministry of Health, Labour and Welfare approved nivolumab in September 2017 for the treatment of unresectable advanced or recurrent GC/GEJC that had progressed following chemotherapy. The latest Japanese guideline for GC recommends nivolumab as a third-line therapy, regardless of HER2 status. Owing to lack of evidence, administering nivolumab as first-line or second-line therapy is presently not recommended.

In the USA, as mentioned earlier, pembrolizumab was approved in patients with treatment-refractory MSI-high solid tumor, and pembrolizumab represents a candidate for the treatment of GC with MSI-high as salvage therapy.

\section{ICI-related clinical features in GC}

Among patients treated with ICIs, a small subset manifests atypical patterns of response including pseudo-progression and hyper-progression of tumor and hyper-response to subsequent chemotherapy. Although the mechanisms of these

Table I Results of Phase III trials of ICls in patients with GC

\begin{tabular}{|c|c|c|c|c|c|c|c|c|c|}
\hline Trial & Line & $\begin{array}{l}\text { Primary } \\
\text { end point }\end{array}$ & $\begin{array}{l}\text { PD-LI } \\
\text { status }\end{array}$ & Arm & $\mathbf{N}$ & $\mathbf{R R}(\%)$ & $\begin{array}{l}\text { PFS } \\
\text { (months) }\end{array}$ & $\begin{array}{l}\text { OS } \\
\text { (months) }\end{array}$ & $\begin{array}{l}\text { Grade } \geq 3 \\
\text { TRAEs (\%) }\end{array}$ \\
\hline ATTRACTION-02 & $\begin{array}{l}\text { Third } \\
\text { and } \\
\text { above }\end{array}$ & OS & Unselected & $\begin{array}{l}\text { Nivolumab } \\
\text { Placebo }\end{array}$ & $\begin{array}{l}330 \\
163\end{array}$ & $\begin{array}{l}11.2 \\
0\end{array}$ & $\begin{array}{l}I .6 \\
I .5 \\
H R=0.60 \\
(0.49-0.75) \\
P<0.000 I\end{array}$ & $\begin{array}{l}7.5 \\
5 . I \\
H R=0.63 \\
(0.49-0.75) \\
P<0.000 I\end{array}$ & $\begin{array}{l}10 \\
4\end{array}$ \\
\hline KEYNOTE-06I & Second & $\begin{array}{l}\text { OS } \\
\text { PFS }\end{array}$ & Positive & $\begin{array}{l}\text { Nivolumab } \\
\text { PTX }\end{array}$ & $\begin{array}{l}196 \\
199\end{array}$ & $\begin{array}{l}16 \\
14\end{array}$ & $\begin{array}{l}1.5 \\
4.1 \\
H R=1.27 \\
(1.03-1.57)\end{array}$ & $\begin{array}{l}9.1 \\
8.3 \\
H R=0.82 \\
(0.66-I .03) \\
P=0.042 I\end{array}$ & $\begin{array}{l}14 \\
35\end{array}$ \\
\hline JAVELIN Gastric 300 & Third $\leqq$ & OS & Unselected & $\begin{array}{l}\text { Nivolumab } \\
\text { Physician's choice } \\
\text { (PTX or IRI) }\end{array}$ & $\begin{array}{l}272 \\
133\end{array}$ & $\begin{array}{l}2.2 \\
4.3\end{array}$ & $\begin{array}{l}I .4 \\
2.7 \\
H R=I .73 \\
(I .4-2.2) \\
P>0.99\end{array}$ & $\begin{array}{l}4.6 \\
5.0 \\
H R=I . I \\
(0.9-I .4) \\
P=0.8 I\end{array}$ & $\begin{array}{l}9.2 \\
31.6\end{array}$ \\
\hline
\end{tabular}

Abbreviations: GC, gastric cancer; ICl, immune checkpoint inhibitor; IRI, irinotecan; OS, overall survival; PD-LI, programmed death ligand I; PFS, progression-free survival; PTX, paclitaxel; RR, response ratio; TRAEs, treatment-related adverse events. 
Table 2 Ongoing trials of $\mathrm{ICls}$ in gastric cancer

\begin{tabular}{l|l|l|l|l|l}
\hline Trial & Phase & Line & Arm & $\begin{array}{l}\text { PD-LI } \\
\text { status }\end{array}$ & $\begin{array}{l}\text { Primary } \\
\text { end point }\end{array}$ \\
\hline CheckMate 649 & III & First & $\begin{array}{l}\text { Nivolumab + ipilimumab nivolumab + XELOX/FOLFOX } \\
\text { XELOX/FOLFOX }\end{array}$ & Unselected & OS in PD-LI+ \\
\hline ATTRACTION-4 & II/III & First & $\begin{array}{l}\text { Nivolumab + SOX/XELOX } \\
\text { SOX/XELOX }\end{array}$ & Unselected & OS and PFS \\
\hline KEYNOTE-062 & III & First & $\begin{array}{l}\text { Pembrolizumab } \\
\text { Pembrolizumab + cisplatin + 5-FU cisplatin +5-FU }\end{array}$ & Positive & OS and PFS \\
\hline JAVELIN Gastric I00 & III & First & $\begin{array}{l}\text { Oxaliplatin + fluoropyrimidine } \rightarrow \text { avelumb (maintenance) } \\
\text { Oxaliplatin + fluoropyrimidine }\end{array}$ & Unselected & OS and PFS \\
\hline NivoRam & I/II & Second & $\begin{array}{l}\text { Nivolumab + ramcirumab } \\
\text { Ramucirumab }\end{array}$ & Unselected & PFS \\
\hline- & I/II & Second & $\begin{array}{l}\text { Nivolumab + ramcirumab + paclitaxel } \\
\text { Ramcirumab + paclitaxel }\end{array}$ & Unselected & PFS \\
\hline ONO-4538-38 & III & Adjuvant & $\begin{array}{l}\text { Nivolumab + S-I/XELOX } \\
\text { S-I/XELOX }\end{array}$ & Unselected & RFS \\
\hline KEYNOTE-585 & III & $\begin{array}{l}\text { Neoadjuvant/ } \\
\text { adjuvant }\end{array}$ & $\begin{array}{l}\text { Pembrolizumab + XP/FP } \\
\text { Placebo + XP/FP }\end{array}$ & Unselected & $\begin{array}{l}\text { OS, RFS, } \\
\text { pathological CR }\end{array}$ \\
\hline
\end{tabular}

Abbreviations: 5-FU, 5-fluorouracil; CR, complete response; FOLFOX, 5-FU plus oxaliplatin; FP, 5FU plus cisplatin; ICl, immune checkpoint inhibitor; OS, overall survival; PD-LI, programmed death ligand I; PFS, progression-free survival; RFS, recurrence-free survival; SOX, S-I plus oxaliplatin; XELOX, capecitabine plus oxaliplatin; XP, capecitabine plus cisplatin.

reactions are not well elucidated, they may affect clinical decisions and the future development of treatment strategies using ICIs. We have reviewed these responses illustrating each of them with cases of patients treated by us.

\section{Pseudo-progression}

The guidelines for the evaluation of immune therapy activity in solid tumors define pseudo-progression as a transient increase in tumor size while undergoing immunotherapy for cancer. ${ }^{29}$ One hypothesis of pseudo-progression is that tumor microenvironment edema and infiltration of immune cells cause a transient increase. Additionally, a delayed clinical response may cause a transient increase.

Pseudo-progression was initially noted in a melanoma while studying the anti-CTLA4 inhibitor ipilimumab. ${ }^{30}$ Later, it was also identified in the anti-PD-1 therapy research involving pembrolizumab and nivolumab. ${ }^{31}$ Unconventional immune response or pseudo-progression was observed in $2.8 \%-15.8 \%$ of patients with melanoma treated with ICIs. ${ }^{32-35}$ Although pseudo-progression with ICIs occurred across various solid tumor types, it has not yet been reported in patients with GC.

We observed one case of pseudo-progression in lung metastasis during treatment with nivolumab. A 79-yearold male was diagnosed with GC in the residual stomach after distal gastrectomy for early GC. CT findings revealed para-aortic lymph node metastasis. The patient was treated with standard chemotherapy; however, the para-aortic lymph node and lung metastases were noted to be enlarged. We initiated nivolumab as third-line therapy for him. We detected a further enlargement of the lung metastatic lesions at the first CT evaluation 6 weeks following the onset of treatment (Figure 1A, B, D, and E). However, provided that his general condition was stable and his total tumor burden remained low, nivolumab was continued. A second CT evaluation at 12 weeks revealed shrinkage in the lung lesions (Figure 1C and F). Lastly, his disease was controlled by nivolumab administration for nearly 1 year.

Pseudo-progression is a clinical challenge for practitioners and patients. When patients are informed regarding potential pseudo-progression, they may continue treatment in the presence of tumor enlargement or new tumor lesions on imaging scans. However, some of these patients have true disease progression and alternative treatment options need to be considered. Since these findings of pseudo-progression would have been classified as "progressive disease" using historic Response Evaluation Criteria in Solid Tumors criteria, new imaging criteria had to be developed for precisely evaluating the immune-related response. Immune-related response criteria (IrRC) ${ }^{29}$ have been introduced, and they require the confirmation of disease progression on repeated, successive scans for at least 4 weeks following the first documented progression. Moreover, these criteria include the measurement of new lesions for assessing the total tumor burden. 

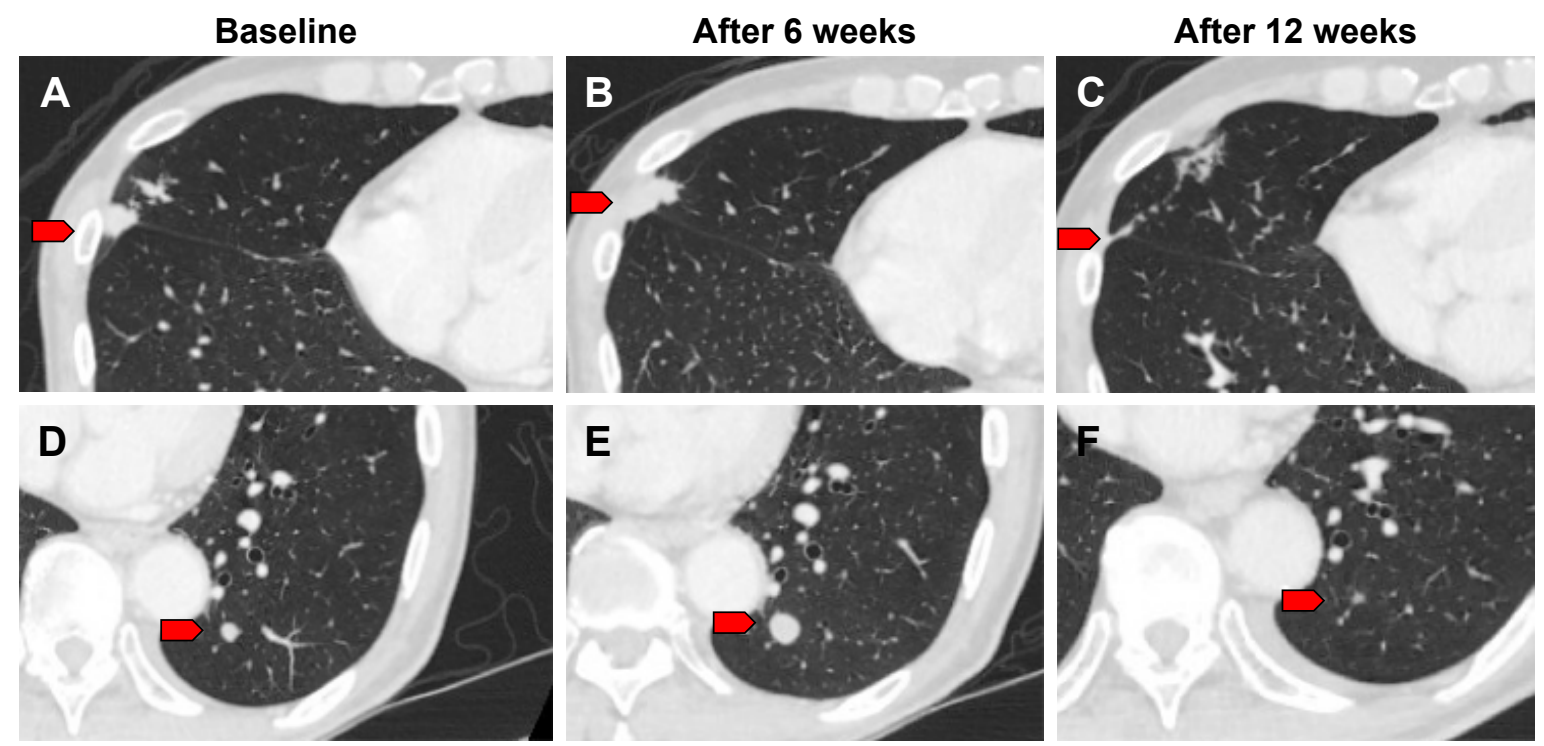

Figure I CT imaging of pseudo-progression.

Notes: (A, D) Representative CT imaging before nivolumab treatment. (B, E) CT evaluation at 6 weeks after onset of nivolumab treatment. (C, F) CT evaluation at 12 weeks revealed shrinkage of these lung lesions. Red arrows indicate lung metastases.

Additional information is necessary for using the IrRC for making treatment-related decisions in GC. No data from the ATTRACTION-2 study have been published regarding pseudo-progression. ${ }^{2}$ Although we experienced one pseudoprogression, most of our cases showed continuous progression following nivolumab administration beyond initial progression. GC often rapidly progresses and causes complications such as bowel obstruction, hemorrhage, and severe ascites. When disease progression is observed, physicians need to carefully evaluate the patient's condition, consider other treatment options, and make a decision regarding the continuation of nivolumab administration.

\section{Hyper-progression}

Hyper-progression in a disease is defined as dramatic progression that outpaces the expected rate of growth in the absence of ICIs. ${ }^{36}$ Hyper-progression has been observed across numerous tumor types, including melanoma, urothelial cancer, CRC, ovarian cancer, biliary tract cancer, lymphoma, non-small-cell lung cancer, and head and neck squamous cell carcinoma. ${ }^{37-39}$ Therefore, hyper-progression is considered independent of tumor histology.

We observed hyper-progression in a 59-year-old male patient initially diagnosed with GC involving HER2 overexpression. He was treated using capecitabine plus cisplatin combined with trastuzumab, paclitaxel plus ramucirumab, and irinotecan monotherapy. After four irinotecan administrations, his liver and lung metastases progressed. We then started him on $3 \mathrm{mg} / \mathrm{kg}$ of nivolumab every 2 weeks as fourth-line therapy. Following two cycles of nivolumab, his liver enzymes increased. We performed CT for distinguishing irAE and disease progression. The CT showed multiple new lesions and remarkable enlargement of liver metastatic lesions, indicating a fast cancer progression (Figure 2). His condition worsened rapidly, and he died a few weeks later.

The mechanism of hyper-progression remains largely unknown. Identification of the predictors and mechanisms of hyper-progression has become essential, so that patients susceptible to develop hyper-progression with ICIs are not treated with any of them and will not be harmed.

Hyper-progression has been associated with the following two clinical variables: older age and regional recurrence in an irradiated field. ${ }^{37,39}$ Moreover, specific genomic alterations such as MDM2 family amplification and EGFR aberrations were reportedly associated with hyper-progression. ${ }^{40}$ Further characterization of hyper-progression is warranted and may provide insights into safer and more efficient administration of immunotherapy.

\section{Hyper-response to subsequent chemotherapy}

Studies have reported that ICIs increased the sensitivity to subsequent chemotherapy for some cancer types (Table 3). ${ }^{41-43}$ In non-small-cell lung cancer, a retrospective analysis showed a 39\% ORR to single-agent chemotherapy administered following cancer progression on anti-PD-1 antibody, exceeding 


\section{Before starting nivolumab}
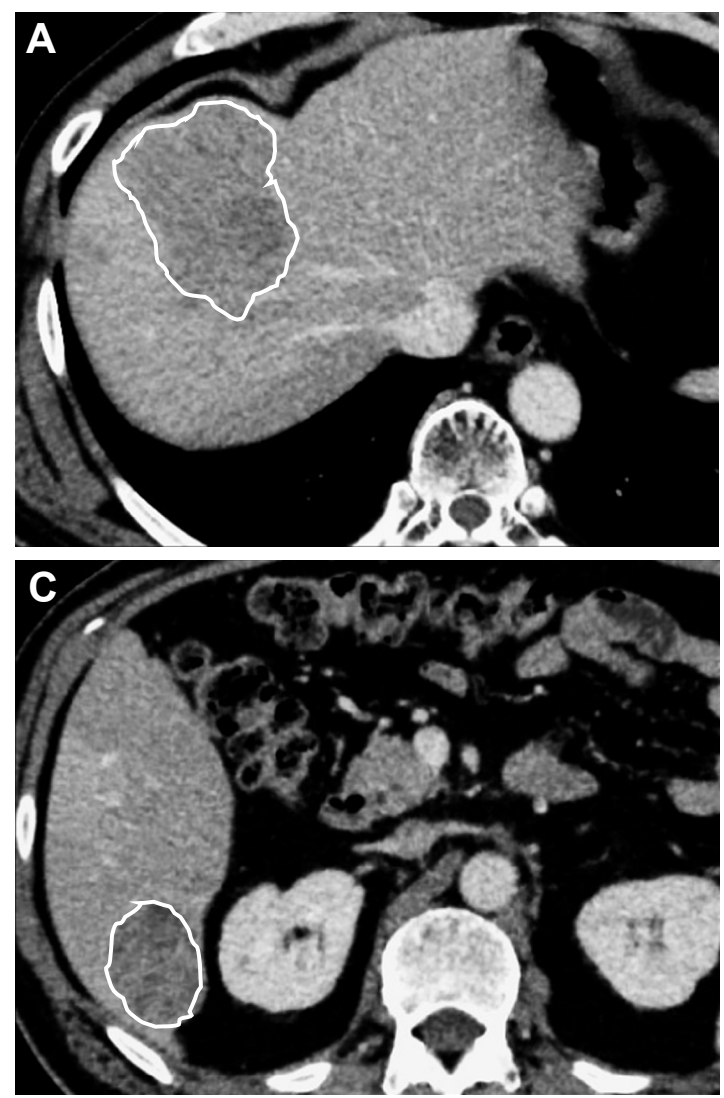

After 3 administrations of nivolumab
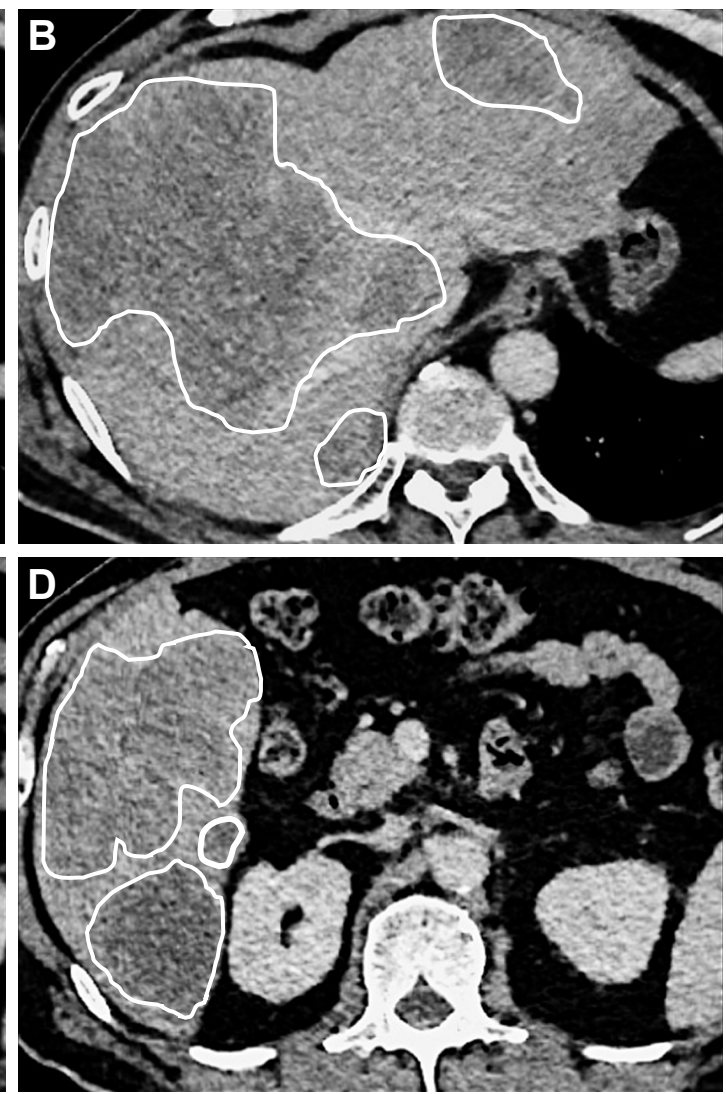

Figure 2 CT imaging of hyper-progression.

Notes: (A, C) Representative CT imaging before nivolumab treatment. (B, D) CT evaluation after three cycles of nivolumab showed remarkable enlargement of liver metastatic lesions, indicating a fast cancer progression. White line shows margins of liver metastases.

the $37 \%$ ORR in first-line chemotherapy. ${ }^{44}$ Another retrospective analysis evaluated ORR in salvage chemotherapy, following ICIs in metastatic urothelial cancer. ${ }^{45}$ The ORR in patients who received ICIs in a first-line therapy followed by chemotherapy was $64 \%$, compared with $50 \%$ for standard platinum-based chemotherapy in the first-line therapy. These findings strongly suggest that ICIs enhance the antitumor effect of subsequent chemotherapy.

Although there is no evidence for the efficacy of chemotherapy following nivolumab administration in GC, we observed two cases with remarkable tumor shrinkage in GC in response to conventional cytotoxic chemotherapy following the progression of the cancer during treatment with nivolumab. Case 1 was a 71-year-old male and case 2 was a 79-year-old male (the same patient with pseudo-progression mentioned earlier). Both patients were administered nivolumab as third-line chemotherapy, and disease progression was observed. As fourth-line chemotherapy, case 1 was treated using irinotecan, and case 2 was treated using oxaliplatin plus capecitabine. Both patients showed a durable response over 12 months in their fourth-line chemotherapy. Figure 3 shows the time course of tumor burden and treatment.

These results show that our patients showed durable responses to salvage-line chemotherapy after the failure of nivolumab treatment. Because salvage-line chemotherapy is expected to present a very limited response in patients with $\mathrm{GC}$, this result indicates that nivolumab may increase the sensitivity to subsequent chemotherapy.

One possible explanation for this effect is that nivolumab remains in the body after discontinuation and works in combination with subsequent chemotherapy. While its half-life is $\sim 2$ weeks, nivolumab occupies the PD-1 receptor of lymphocytes for more than 2 months. ${ }^{46}$ The bound nivolumab at the time of subsequent chemotherapy possibly adds a therapeutic effect. In fact, in non-small-cell lung cancer, a better response can be obtained by chemotherapy combined with ICI. ${ }^{47}$ Another possibility is the modification of the 
Table 3 Response to chemotherapy after ICls as reported in the literature

\begin{tabular}{|c|c|c|c|c|c|c|c|}
\hline References & $\begin{array}{l}\text { Age } \\
\text { (years) }\end{array}$ & Sex & Diagnosis & $\begin{array}{l}\text { Chemotherapy prior } \\
\text { to ICls }\end{array}$ & ICls & $\begin{array}{l}\text { Chemotherapy } \\
\text { after ICls }\end{array}$ & $\begin{array}{l}\text { Response to } \\
\text { chemotherapy } \\
\text { after ICls }\end{array}$ \\
\hline \multirow[t]{6}{*}{$\begin{array}{l}\text { Dwary et al, } \\
2017^{41}\end{array}$} & 61 & $\mathrm{~F}$ & HNSCC & First line: CDDP + RT & Pembrolizumab & $\begin{array}{l}\text { CBDCA + PTX + } \\
\text { cetuximab }\end{array}$ & $C R$ \\
\hline & 54 & $M$ & HNSCC & $\begin{array}{l}\text { First line: } C D D P+R T \\
\text { Second line: } C B D C A+5 \\
\text { FU + cetuximab } \\
\text { Third line: } M T X\end{array}$ & Pembrolizumab & $\begin{array}{l}\text { CBDCA + PTX + } \\
\text { cetuximab }\end{array}$ & $C R$ \\
\hline & 54 & M & HNSCC & $\begin{array}{l}\text { First line: DTX + CDDP } \\
\text { Second line: CBDCA + } \\
\text { PTX + RT }\end{array}$ & Pembrolizumab & $\begin{array}{l}\text { CBDCA + PTX + } \\
\text { cetuximab }\end{array}$ & PR (>2 months) \\
\hline & 60 & $\mathrm{~F}$ & NSCLC & $\begin{array}{l}\text { First line: } \text { CBDCA + PEM + } \\
\text { bevacizumab } \\
\text { Second line: DTX }\end{array}$ & Nivolumab & DTX & $C R$ \\
\hline & 50 & M & NSCLC & First line: CBDCA + PEM & Nivolumab & DTX & PR (>2 months) \\
\hline & 21 & M & $\begin{array}{l}\text { T-cell rich B-cell } \\
\text { lymphoma }\end{array}$ & First line: R-CHOP & Nivolumab & $\begin{array}{l}\text { GEM + prednisone + } \\
\text { CDDP }\end{array}$ & $\mathrm{CR}$ \\
\hline \multirow[t]{2}{*}{$\begin{array}{l}\text { Ogawara et al, } \\
2018^{42}\end{array}$} & 66 & $\mathrm{~F}$ & NSCLC & $\begin{array}{l}\text { First line: CDDP + GEM } \\
\text { Second line: DTX }\end{array}$ & Nivolumab & S-I & PR (5 months) \\
\hline & 75 & M & NSCLC & $\begin{array}{l}\text { First line: CDDP + PEM } \\
\text { Second line: DTX } \\
\text { Third line: S-I }\end{array}$ & Nivolumab & CBDCA + nab-PTX & PR (5 months) \\
\hline $\begin{array}{l}\text { Simon et al, } \\
2017^{43}\end{array}$ & 56 & $M$ & Melanoma & $\begin{array}{l}\text { First line: vemurafenib } \\
\text { Second line: dabrafenib + } \\
\text { trametinib } \\
\text { Third line: MTX }\end{array}$ & $\begin{array}{l}\text { Fourth line: } \\
\text { ipilimumab } \\
\text { Fifth line: nivolumab }\end{array}$ & $\begin{array}{l}\text { Dacarbazine }+ \\
\text { CDDP }\end{array}$ & PR (>3 months) \\
\hline \multirow[t]{2}{*}{ Present cases } & 71 & $M$ & Gastric cancer & $\begin{array}{l}\text { First line: CDDP + } \\
\text { capecitabine }+ \text { trastuzumab } \\
\text { Second line: PTX + Rmab }\end{array}$ & Nivolumab & Irinotecan & PR ( $>12$ months) \\
\hline & 79 & M & Gastric cancer & $\begin{array}{l}\text { First line: CDDP + S-I + RT } \\
\text { Second line: PTX }\end{array}$ & Nivolumab & $\begin{array}{l}\text { Capecitabine + } \\
\text { oxaliplatin }\end{array}$ & PR ( $>12$ months) \\
\hline
\end{tabular}

Abbreviations: 5-FU, 5-fluorouracil; CBDCA, carboplatin; CDDP, cisplatin; CR, complete response; DTX, docetaxel; GEM, gemcitabine; ICI, immune checkpoint inhibitor; HNSCC, head and neck squamous cell carcinoma; MTX, methotrexate; nab-PTX, albumin-bound PTX; NSCLC, non-small-cell lung cancer; PEM, pemetrexed; PR, partial response; R-CHOP, rituximab, cyclophosphamide, doxorubicin, and vincristine; Rmab, ramucirumab; RT, radiation therapy.
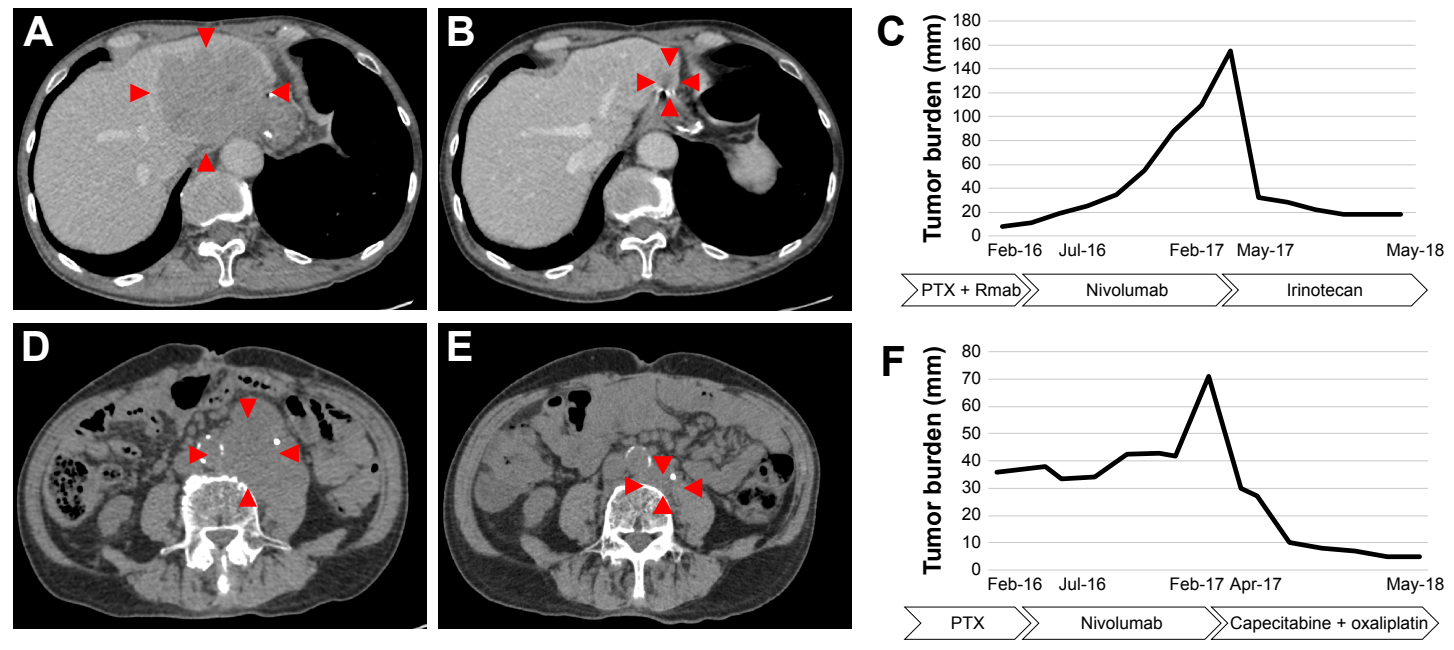

Figure 3 CT imaging and clinical course of the hyper-response in subsequent therapy.

Notes: (A) Representative CT imaging of case I after nivolumab treatment. Liver metastasis progressed to $90 \mathrm{~mm}$. (B) CT imaging showed significant response to irinotecan. Liver metastasis has shrunken to $20 \mathrm{~mm}$. (C) Time course of case I, including tumor burden and treatment. Tumor burden, shown as a line graph, was calculated as the sum of the target lesions according to the Response Evaluation Criteria in Solid Tumors, version I.I. (D) Representative CT imaging of case 2 after progression on nivolumab. Para-aortic lymph node metastasis progressed to $60 \mathrm{~mm}$. (E) CT imaging showed remarkable response to oxaliplatin and capecitabine. (F) Time course of case 2, including tumor burden and treatment. Red markers show the margin of tumor.

Abbreviations: PTX, paclitaxel; Rmab, ramucirumab. 
tumor microenvironment by nivolumab enhances the cytotoxic effect of subsequent therapy. Exposure to nivolumab could change the microenvironment by an increased pool of activated $\mathrm{T}$ cells that would then increase the sensitivity to chemotherapy. Further large-scale studies are warranted for evaluating the efficacy and safety of chemotherapy following immunotherapy and for identifying patients who may benefit from this. The cases reported in this review suggest that nivolumab may increase the sensitivity to subsequent chemotherapy in GC.

\section{Biomarkers for evaluating $\mathbf{I C I}$ benefits in GC}

Immunotherapy has changed the therapeutic strategy for patients with GC and has improved OS and clinical responses. Unfortunately, the response rate remains low, and the predictive factors that will identify the subgroup of patients who derive the greater benefit of therapy should be determined. Any biomarker that could be applied as part of standard therapeutic decision-making in GC has not been identified to date. In this section, we have reviewed the present scenario of biomarker for evaluating ICI benefits with emphasis on GC.

\section{Programmed death ligand I}

PD-1 is a negative co-stimulatory receptor that is primarily expressed on activated T cells. ${ }^{48}$ The PD- 1 receptor is an inhibitory $\mathrm{T}$-cell receptor primarily engaged within the tumor microenvironment by its two known ligands, PD-L1 and programmed cell death 1 ligand 2 (PD-L2). PD-L1 is normally expressed by a subset of macrophages and can be induced on activated lymphocytes, endothelial cells, and other non-malignant cell types in an inflammatory microenvironment. It forms part of a physiological process to down-modulate ongoing host immune responses in peripheral tissues. ${ }^{49}$ However, tumor cells and associated stromal cells can also express PD-L1, thereby turning off T-cell activation and allowing uncontrolled tumor cell proliferation. Therefore, PD-L1 expression has been considered to be one of the most promising biomarkers for anti-PD-1 drugs. ${ }^{50}$

PD-L1 is expressed in $\sim 25 \%-65 \%$ of GC tissues, whereas it is undetectable in normal gastric mucosa of healthy individuals. ${ }^{51-53}$ In KEYNOTE-061, PD-L1 expression has been correlated with better treatment outcome with pembrolizumab. ${ }^{14}$ These data reinforce the utility of PD-L1 expression for selecting patients for treatment with pembrolizumab monotherapy. On the contrary, the data from the ATTRACTION-2 study showed a significant benefit of nivolumab in all patients, including those with PD-L1-negative tumors. However, PD-L1 expression in ATTRACTION-2 was retrospectively assessed on tumor cells using the 28-8 pharmDx assay, with PD-L1 expression available for only $39 \%$ of the patients. ${ }^{2}$ In contrast, PD-L1 expression was prospectively assessed on tumor cells and tumor-associated lymphocytes and macrophages using the 22C3 pharmDx assay in KEYNOTE-61, which may be more efficient in predicting outcomes than tumor PD-L1 expression alone. PD-L1 expression could possibly function as a biomarker for identifying patients who are likely to benefit the most from these therapies, keeping in mind that existing data support the clinical benefit of ICIs even in PD-L1negative patients. Overall, PD-L1 expression would not be sufficient as a biomarker for identifying patients who may be responsive to anti-PD-1 therapy.

\section{Tumor-infiltrating lymphocytes (TILs)}

The presence of TILs can be detected in various cancers, including GC. TILs are considered as a selected population of T-cells with specific immunological reactivity against tumor cell. Therefore, the absence of TILs may contribute to immunotherapy resistance. ${ }^{54}$ Specifically, the density of tumor infiltrated with $\mathrm{CD} 8^{+} \mathrm{T}$ cells has been correlated with a response to anti-PD1 treatment in the anti-PD1 therapy for melanoma. ${ }^{55}$ Correlations between the levels of TILs and clinical outcome have been reported. ${ }^{56} \mathrm{~A}$ strong lymphocytic infiltration is associated with a good clinical outcome in numerous tumor types, including melanoma, head and neck cancer, breast cancer, bladder cancer, urothelial cancer, ovarian cancer, CRC, renal cancer, prostatic cancer, and lung cancer. However, the association between TILs and ICIs has not yet been reported in GC.

\section{Microsatellite instability}

Defects in DNA mismatch repair result in tumors with an increased number of somatic mutations that can induce an innate antitumor immune response and stimulate tumors for becoming more responsive to immune checkpoint blockade. ${ }^{57}$ As previously mentioned, on the basis of data from the Phase I and Phase II studies, ${ }^{16-20}$ pembrolizumab was approved by the US FDA for any solid tumors with MSI-high in May 2017.

Some GCs have a high mutational burden, particularly MSI-high tumors. The Cancer Genome Atlas has categorized GC into the following four molecular subtypes: Epstein-Barr virus (EBV)-positive, MSI-high, genomically stable, and chromosomally instable. ${ }^{58}$ Among these, MSI-high accounted for $22 \%$ of the patients with GC. 
In a post hoc exploratory analysis of KEYNOTE-061, patients whose tumors had high levels of MSI were retrospectively assessed at a central laboratory, irrespective of PD-L1 CPS. ${ }^{15}$ The results showed a particularly large treatment effect with pembrolizumab. Recently, Kim et al performed molecular characterization of tissues from 61 patients with GC who were treated with pembrolizumab as salvage treatment in a prospective Phase II clinical trial. ${ }^{59}$ In patients with MSI-high tumors, responses were observed in $85.7 \%$ of the patients, which suggests that these GC subtypes are particularly responsive to anti-PD-1 therapy.

\section{Epstein-Barr virus}

Integrated oncogenic viruses represent another genetic alteration in cancers that confers neo-antigenicity, thus serving as a molecular biomarker predictive of response to immune checkpoint blockade. Integrated viruses expressing oncogenes drive several human cancers in both immunodeficient and immunocompetent individuals. ${ }^{50} \mathrm{EBV}$-positive GCs are characterized by marked intra- or peri-tumoral immune cell infiltration and often exhibits the genomic amplification of the chromosome 9 locus containing genes encoding PD-L1 and PD-L2. ${ }^{60}$ Prior clinical trials have shown that EBV-positive tumors exhibit robust PD-L1 expression both in cancer cells and immune cells. ${ }^{13,61}$ Notably, Kim et al reported that in 61 patients with GC who were treated with pembrolizumab as salvage treatment, ORR was $100 \%$ in EBV-positive tumors. Remarkably, all six patients with EBV-positive GC achieved PR with a median duration of response of 8.5 months in third-line therapy. This suggests that the presence of EBV represents a powerful predictive biomarker for immune checkpoint blockade efficacy in GC.

\section{ctDNA}

Plasma-derived ctDNA sequencing has been shown to reproduce tumor tissue exome sequencing for identifying patients who are likely to respond to pembrolizumab. ${ }^{62} \mathrm{Kim}$ et al showed that ctDNA mutational load score was well correlated with response to pembrolizumab, and it appeared to predict PFS, at least, as well as the tissue mutational load. ${ }^{59}$ These data suggest that in patients unable or unwilling to undergo invasive tissue biopsy, broad ctDNA profiling may suffice to accurately identify potential candidates for pembrolizumab therapy. However, this approach will fail to identify EBVpositive tumors that generally exhibit a low mutational load. Post-treatment changes in ctDNA were also predictors of both response and progression in GC. The identification of patients with a high risk of progression on the basis of changes in ctDNA at an early stage is useful for identifying patients who may need alternative therapeutic approaches and prevent confusion caused by pseudo-progression.

\section{Gut microbiota}

Specific members of the gut microbiota act as modulators of TILs and may influence the efficacy of immunotherapy. Bifidobacterium spp. enhanced the efficacy of anti-PD-L1 therapy in a mouse model. ${ }^{63}$ Oral administration of Bifidobacterium alone improved tumor control to the same degree as PD-L1-specific antibody therapy, and anti-PD-L1 combination treatment nearly abolished tumor outgrowth. Similarly, Bifidobacterium spp. reportedly enhanced the efficacy of cancer immunotherapy by CTLA. ${ }^{64}$

A recent report demonstrated that primary resistance to ICIs can be attributed to abnormal gut microbiome composition. ${ }^{65}$ In patients with advanced cancer, antibiotics inhibit the clinical benefit of ICIs. Fecal microbiota transplantation (FMT) from cancer patients, who responded to ICIs, into sterile mice enhanced the antitumor effects of PD-1 blockade, whereas FMT from non-responders did not. The correlation between the gut microbiome of melanoma patients undergoing anti-PD-1 therapy and its efficacy has also been reported. ${ }^{66}$ Significant differences were observed in the diversity and composition of the gut microbiome of responders vs non-responders. The analysis of patient fecal microbiome samples in responding patients showed significantly higher alpha diversity $(P<0.01)$ and relative abundance of bacteria of the Ruminococcaceae family $(P<0.01)$. These results indicate that manipulating the microbiota modulates cancer immunotherapy.

\section{Conclusion}

In the era of targeted therapy and personalized medicine, immunotherapy has shifted the treatment paradigm of GC. Although nivolumab has proven its efficacy as monotherapy in the salvage-line setting, further research is required for determining other regimens or combinations that will improve efficacy and tolerability. In addition, for guiding patient selection and identifying those who could benefit from ICIs, deeper genetic and immunological characterization of $\mathrm{GC}$ is required.

\section{Disclosure}

The authors report no conflicts of interest in this work.

\section{Acknowledgement}

The authors thank Yasuo Hamamoto, Keio University Cancer Center for their generous support. 


\section{References}

1. Ferlay J, Soerjomataram I, Dikshit R, et al. Cancer incidence and mortality worldwide: sources, methods and major patterns in GLOBOCAN 2012. Int J Cancer. 2015;136(5):E359-E386.

2. Kang YK, Boku N, Satoh T, et al. Nivolumab in patients with advanced gastric or gastro-oesophageal junction cancer refractory to, or intolerant of, at least two previous chemotherapy regimens (ONO-4538-12, ATTRACTION-2): a randomised, double-blind, placebo-controlled, phase 3 trial. Lancet. 2017;390(10111):2461-2471.

3. Janjigian YY OP, Calvo E, et al. Nivolumab \pm ipilimumab in pts with advanced $(\mathrm{adv}) /$ metastatic chemotherapy-refractory (CTx-R) gastric $(G)$, esophageal (E), or gastroesophageal junction (GEJ) cancer: CheckMate 032 study. Paper presented at: ASCO General Meeting; June 2-4, 2017; Chicago, IL.

4. Janjigian YY, Bendell J, Calvo E, et al. CheckMate-032 study: efficacy and safety of nivolumab and nivolumab plus ipilimumab in patients with metastatic esophagogastric cancer. J Clin Oncol. 2018; 36(28):2836-2844.

5. Michot JM, Bigenwald C, Champiat S, et al. Immune-related adverse events with immune checkpoint blockade: a comprehensive review. Eur J Cancer. 2016;54:139-148.

6. Borghaei H, Paz-Ares L, Horn L, et al. Nivolumab versus docetaxel in advanced nonsquamous non-small-cell lung cancer. $N$ Engl J Med. 2015;373(17):1627-1639.

7. Brahmer J, Reckamp KL, Baas P, et al. Nivolumab versus docetaxel in advanced squamous-cell non-small-cell lung cancer. $N$ Engl J Med. 2015;373(2):123-135.

8. Carbone DP, Reck M, Paz-Ares L, et al. First-line nivolumab in stage IV or recurrent non-small-cell lung cancer. $N$ Engl J Med. 2017;376(25):2415-2426.

9. Ferris RL, Blumenschein G, Fayette J, Jr, et al. Nivolumab for recurrent squamous-cell carcinoma of the head and neck. $N$ Engl J Med. 2016;375(19):1856-1867.

10. Larkin J, Minor D, D'Angelo S, et al. Overall survival in patients with advanced melanoma who received nivolumab versus investigator's choice chemotherapy in CheckMate 037: a randomized, controlled, open-label Phase III trial. J Clin Oncol. 2018;36(4):383-390.

11. Motzer RJ, Escudier B, McDermott DF, et al. Nivolumab versus everolimus in advanced renal-cell carcinoma. N Engl J Med. 2015;373(19): 1803-1813.

12. Robert C, Long GV, Brady B, et al. Nivolumab in previously untreated melanoma without BRAF mutation. N Engl J Med. 2015;372(4):320-330.

13. Muro K, Chung HC, Shankaran V, et al. Pembrolizumab for patients with PD-L1-positive advanced gastric cancer (KEYNOTE-012): a multicentre, open-label, phase 1b trial. Lancet Oncol. 2016;17(6): 717-726.

14. Fuchs CS, Doi T, Jang RWJ, et al. KEYNOTE-059 cohort 1: efficacy and safety of pembrolizumab (pembro) monotherapy in patients with previously treated advanced gastric cancer. J Clin Oncol. 2017;35(15 Suppl):4003.

15. Shitara K, Özgüroğlu M, Bang YJ, et al. Pembrolizumab versus paclitaxel for previously treated, advanced gastric or gastro-oesophageal junction cancer (KEYNOTE-061): a randomised, open-label, controlled, phase 3 trial. Lancet. 2018;392(10142):123-133.

16. Le DT, Durham JN, Smith KN, et al. Mismatch repair deficiency predicts response of solid tumors to PD-1 blockade. Science. 2017; 357(6349):409-413.

17. Dt L, Kavan P, Kim TW. KEYNOTE-164: pembrolizumab for patients with advanced microsatellite instability high (MSI-H) colorectal cancer. J Clin Oncol. 2018;36(15 Suppl):3514.

18. Plimack ER, Bellmunt J, Gupta S, et al. Safety and activity of pembrolizumab in patients with locally advanced or metastatic urothelial cancer (KEYNOTE-012): a non-randomised, open-label, phase 1b study. Lancet Oncol. 2017;18(2):212-220.

19. Hansen AR, Massard C, Ott PA, et al. Pembrolizumab for advanced prostate adenocarcinoma: findings of the KEYNOTE-028 study. Ann Oncol. 2018;29(8):1807-1813.
20. Schellens JHM, Marabelle A, Zeigenfuss S, Ding J, Pruitt SK, Chung HC. Pembrolizumab for previously treated advanced cervical squamous cell cancer: preliminary results from the phase 2 KEYNOTE-158 study. $J$ Clin Oncol. 2017;35(15 Suppl):5514.

21. Bang YJ, Ruiz EY, Van Cutsem E, et al. Phase 3, randomised trial of avelumab versus physician's choice of chemotherapy as third-line treatment for patients with advanced gastric or gastro-oesophageal junction cancer: primary analysis of JAVELIN Gastric 300. Ann Oncol. Epub 2018 Jul 24.

22. Taieb J, Bartolomet M, Cubillo A, et al. JAVELIN Gastric 100: Phase 3 trial of avelumab (anti-PD-L1 antibody) maintenance therapy vs continuation of first-line chemotherapy in patients with unresectable, locally advanced or metastatic gastric or gastroesophageal junction cancer. Ann Oncol. 2016;27(Suppl 2):ii81-ii82.

23. Tabernero J, Bang Y-J, Fuchs CS, et al. KEYNOTE-062: Phase III study of pembrolizumab (MK-3475) alone or in combination with chemotherapy versus chemotherapy alone as first-line therapy for advanced gastric or gastroesophageal junction (GEJ) adenocarcinoma. $J$ Clin Oncol. 2016;34(4 Suppl):TPS185.

24. Janjigian YY, Adenis A, Aucoin J-S, et al. Checkmate 649: a randomized, multicenter, open-label, phase 3 study of nivolumab (Nivo) plus ipilimumab (Ipi) versus oxaliplatin plus fluoropyrimidine in patients (Pts) with previously untreated advanced or metastatic gastric (G) or gastroesophageal junction (GEJ) cancer. J Clin Oncol. 2017;35(4 Suppl):TPS213.

25. Kang Y, Kato K, Chung HC, et al. 671PInterim safety and clinical activity of nivolumab (Nivo) in combination with S-1/capecitabine plus oxaliplatin in patients (pts) with previously untreated unresectable advanced or recurrent gastric/gastroesophageal junction (G/GEJ) cancer: part 1 study of ATTRACTION-04 (ONO-4538-37). Ann Oncol. 2017;28(Suppl 5):v209-v268.

26. Takahari D, Shoji H, Hara H, et al. Preliminary result of phase $1 / 2$ study of ramucirumab plus nivolumab in patients with previously treated advanced gastric adenocarcinoma (NivoRam study). J Clin Oncol. 2018;36(15 Suppl):4047.

27. Wilke H, Muro K, Van Cutsem E, et al. Ramucirumab plus paclitaxel versus placebo plus paclitaxel in patients with previously treated advanced gastric or gastro-oesophageal junction adenocarcinoma (RAINBOW): a double-blind, randomised phase 3 trial. Lancet Oncol. 2014;15(11):1224-1235.

28. Makiyama A, Sagara K, Kawada J, et al. A randomized phase II study of weekly paclitaxel \pm trastuzumab in patients with HER2-positive advanced gastric or gastro-esophageal junction cancer refractory to trastuzumab combined with fluoropyrimidine and platinum: WJOG7112G (T-ACT). J Clin Oncol. 2018;36(15 Suppl):4011.

29. Wolchok JD, Hoos A, O'Day S, et al. Guidelines for the evaluation of immune therapy activity in solid tumors: immune-related response criteria. Clin Cancer Res. 2009;15(23):7412-7420.

30. Wolchok JD, Saenger Y. The mechanism of anti-CTLA-4 activity and the negative regulation of T-cell activation. Oncologist. 2008; 13(Suppl 4):2-9.

31. Chiou VL, Burotto M. Pseudoprogression and immune-related response in solid tumors. J Clin Oncol. 2015;33(31):3541-3543.

32. Hodi FS, Oble DA, Drappatz J, et al. CTLA-4 blockade with ipilimumab induces significant clinical benefit in a female with melanoma metastases to the CNS. Nat Clin Pract Oncol. 2008;5(9):557-561.

33. Millward M, Underhill C, Lobb S, et al. Phase I study of tremelimumab (CP-675 206) plus PF-3512676 (CPG 7909) in patients with melanoma or advanced solid tumours. Br J Cancer. 2013;108(10):1998-2004.

34. Ribas A, Chesney JA, Gordon MS, et al. Safety profile and pharmacokinetic analyses of the anti-CTLA4 antibody tremelimumab administered as a one hour infusion. J Transl Med. 2012;10(1):236.

35. Ribas A, Wolchok JD, Robert C, et al. Updated clinical efficacy of the anti-PD-1 monoclonal antibody pembrolizumab (MK-3475) in 411 patients with melanoma. Paper presented at: 7th Annual Asian Oncology Summit/11th Annual Conference of the Organisationfor-Oncology-and-Translational-Research April 10-12, 2015; Shanghai. 
36. Wang Q, Gao J, Wu X. Pseudoprogression and hyperprogression after checkpoint blockade. Int Immunopharmacol. 2018;58:125-135.

37. Champiat S, Dercle L, Ammari S, et al. Hyperprogressive disease is a new pattern of progression in cancer patients treated by anti-PD-1/ PD-L1. Clin Cancer Res. 2017;23(8):1920-1928.

38. Chubachi S, Yasuda H, Irie H, et al. A case of non-small cell lung cancer with possible "Disease Flare" on nivolumab treatment. Case Rep Oncol Med. 2016;2016:1075641-1075643.

39. Saâda-Bouzid E, Defaucheux C, Karabajakian A, et al. Hyperprogression during anti-PD-1/PD-L1 therapy in patients with recurrent and/or metastatic head and neck squamous cell carcinoma. Ann Oncol. 2017; 28(7):1605-1611.

40. Kato S, Goodman A, Walavalkar V, Barkauskas DA, Sharabi A, Kurzrock R. Hyperprogressors after immunotherapy: analysis of genomic alterations associated with accelerated growth rate. Clin Cancer Res. 2017;23(15):4242-4250.

41. Dwary AD, Master S, Patel A, et al. Excellent response to chemotherapy post immunotherapy. Oncotarget. 2017;8(53):91795-91802.

42. Ogawara D, Soda H, Iwasaki K, et al. Remarkable response of nivolumab-refractory lung cancer to salvage chemotherapy. Thorac Cancer. 2018;9(1):175-180.

43. Simon A, Kourie HR, Kerger J. Is there still a role for cytotoxic chemotherapy after targeted therapy and immunotherapy in metastatic melanoma? A case report and literature review. Chin J Cancer. 2017; 36(1):10.

44. Schvartsman G, Peng SA, Bis G, et al. Response rates to single-agent chemotherapy after exposure to immune checkpoint inhibitors in advanced non-small cell lung cancer. Lung Cancer. 2017;112:90-95.

45. Szabados B, van Dijk N, Tang YZ, et al. Response rate to chemotherapy after immune checkpoint inhibition in metastatic urothelial cancer. Eur Urol. 2018;73(2):149-152.

46. Brahmer JR, Drake CG, Wollner I, et al. Phase I study of single-agent anti-programmed death-1 (MDX-1106) in refractory solid tumors: safety, clinical activity, pharmacodynamics, and immunologic correlates. J Clin Oncol. 2010;28(19):3167-3175.

47. Langer CJ, Gadgeel SM, Borghaei H, et al. Carboplatin and pemetrexed with or without pembrolizumab for advanced, non-squamous nonsmall-cell lung cancer: a randomised, phase 2 cohort of the open-label KEYNOTE-021 study. Lancet Oncol. 2016;17(11):1497-1508.

48. Ribas A. Tumor immunotherapy directed at PD-1. $N$ Engl J Med. 2012;366(26):2517-2519.

49. Dong H, Strome SE, Salomao DR, et al. Tumor-associated B7-H1 promotes T-cell apoptosis: a potential mechanism of immune evasion. Nat Med. 2002;8(8):793-800.

50. Topalian SL, Taube JM, Anders RA, Pardoll DM. Mechanism-driven biomarkers to guide immune checkpoint blockade in cancer therapy. Nat Rev Cancer. 2016;16(5):275-287.

51. Zhang M, Dong Y, Liu H, et al. The clinicopathological and prognostic significance of PD-L1 expression in gastric cancer: a meta-analysis of 10 studies with 1,901 patients. Sci Rep. 2016;6(1):37933.
52. Yuan J, Zhang J, Zhu Y, et al. Programmed death-ligand-1 expression in advanced gastric cancer detected with RNA in situ hybridization and its clinical significance. Oncotarget. 2016;7(26):39671-39679.

53. Kawazoe A, Kuwata T, Kuboki Y, et al. Clinicopathological features of programmed death ligand 1 expression with tumor-infiltrating lymphocyte, mismatch repair, and Epstein-Barr virus status in a large cohort of gastric cancer patients. Gastric Cancer. 2017;20(3):407-415.

54. Woo SR, Corrales L, Gajewski TF. The STING pathway and the T cell-inflamed tumor microenvironment. Trends Immunol. 2015; 36(4):250-256.

55. Tumeh PC, Harview CL, Yearley JH, et al. PD-1 blockade induces responses by inhibiting adaptive immune resistance. Nature. 2014; 515(7528):568-571.

56. Fridman WH, Pagès F, Sautès-Fridman C, Galon J. The immune contexture in human tumours: impact on clinical outcome. Nat Rev Cancer. 2012;12(4):298-306.

57. Chang L, Chang M, Chang HM, Chang F. Microsatellite instability: a predictive biomarker for cancer immunotherapy. Appl Immunohistochem Mol Morphol. 2018;26(2):e15-e21.

58. Cancer Genome Atlas Research Network. Comprehensive molecular characterization of gastric adenocarcinoma. Nature. 2014;513(7517): 202-209.

59. Kim ST, Cristescu R, Bass AJ, et al. Comprehensive molecular characterization of clinical responses to PD-1 inhibition in metastatic gastric cancer. Nat Med. 2018;24(9):1449-1458.

60. Derks S, Liao X, Chiaravalli AM, et al. Abundant PD-L1 expression in Epstein-Barr virus-infected gastric cancers. Oncotarget. 2016;7(22): 32925-32932.

61. Seiwert TY, Burtness B, Mehra R, et al. Safety and clinical activity of pembrolizumab for treatment of recurrent or metastatic squamous cell carcinoma of the head and neck (KEYNOTE-012): an open-label, multicentre, phase 1b trial. Lancet Oncol. 2016;17(7):956-965.

62. Diaz LA, Bardelli A. Liquid biopsies: genotyping circulating tumor DNA. J Clin Oncol. 2014;32(6):579-586.

63. Sivan A, Corrales L, Hubert N, et al. Commensal Bifidobacterium promotes antitumor immunity and facilitates anti-PD-L1 efficacy. Science. 2015;350(6264):1084-1089.

64. Vétizou M, Pitt JM, Daillère R, et al. Anticancer immunotherapy by CTLA-4 blockade relies on the gut microbiota. Science. 2015; 350(6264):1079-1084.

65. Routy B, Le Chatelier E, Derosa L, et al. Gut microbiome influences efficacy of PD-1-based immunotherapy against epithelial tumors. Science. 2018;359(6371):91-97.

66. Gopalakrishnan V, Spencer CN, Nezi L, et al. Gut microbiome modulates response to anti-PD-1 immunotherapy in melanoma patients. Science. 2018;359(6371):97-103.
OncoTargets and Therapy

\section{Publish your work in this journal}

OncoTargets and Therapy is an international, peer-reviewed, open access journal focusing on the pathological basis of all cancers, potential targets for therapy and treatment protocols employed to improve the management of cancer patients. The journal also focuses on the impact of management programs and new therapeutic agents and protocols on
Dovepress

patient perspectives such as quality of life, adherence and satisfaction The manuscript management system is completely online and includes a very quick and fair peer-review system, which is all easy to use. Visit http://www.dovepress.com/testimonials.php to read real quotes from published authors. 